\title{
Cost-Effectiveness of PBO versus Conventional Long-Lasting Insecticidal Bed Nets in Preventing Symptomatic Malaria in Nigeria: Results of a Pragmatic Randomized Trial
}

\author{
Donald S. Shepard, ${ }^{1 \star}$ John U. Odumah, ${ }^{1}$ and Samson T. Awolola ${ }^{2}$ \\ ${ }^{1}$ Heller School for Social Policy and Management, Brandeis University, Waltham, Massachusetts; ${ }^{2}$ Molecular Entomology and Vector Control \\ Research Laboratory, Nigerian Institute of Medical Research, Yaba Lagos, Nigeria
}

\begin{abstract}
Insecticide treated nets (ITNs) have been the major tool in halving malaria's burden since 2000, but pyrethroid insecticide resistance threatens their ongoing effectiveness. In 2017 , the WHO concluded that long-lasting ITNs (LLINs) with a synergist, piperonyl butoxide (PBO), provided additional public health benefit over conventional (pyrethroidonly) LLINs alone in areas of moderate insecticide resistance and endorsed them as a new class of vector control products. We performed an economic appraisal of PBO nets compared with conventional LLINs in 2019 US\$ from prevention and health systems perspectives (including treatment cost offsets). We used data from a pragmatic randomized 2012-2014 trial in Nigeria with epidemiological outcomes in an area with confirmed pyrethroid resistance. Each village had 50 months of epidemiologic data, analyzed by village by month, using negative binomial regression. Compared with LLINs, although adding $\$ 0.90$ per net delivered, PBO nets reduced symptomatic malaria cases by $33.4 \%(95 \%$ $\mathrm{Cl} 10.2-50.6 \%)$. From a prevention perspective, the incremental cost-effectiveness ratio was $\$ 11$ (95\% Cl \$8-\$37) per disability-adjusted life year averted. From the health systems perspective, PBO nets were significantly cost-saving relative to conventional LLINs. The benefit-cost analysis found that the added economic benefits of PBO nets over LLINs were \$201 (95\% CI \$61-\$304) for every \$1 in incremental costs. Growing pyrethroid resistance is likely to strengthen the economic value of PBO nets over LLINs. Beyond their contribution to reducing malaria, PBO nets deliver outstanding economic returns for a small additional cost above conventional LLINs in locations with insecticide resistance.
\end{abstract}

\section{INTRODUCTION}

Over the past two decades, global efforts have made considerable progress in controlling malaria. From 2015 to 2018, 31 malaria-endemic countries were on track to reduce incidence by $40 \%$ or more by $2020 .^{1-4}$ Nevertheless, in 2018 , progress had stalled in many regions and the world still experienced 228 million cases. ${ }^{4}$ Thus, the application of better control measures remains a global priority.

Insecticide-treated nets (ITNs) have been one of the highly effective tools in malaria prevention. ${ }^{5}$ In $2018,50 \%$ of residents of sub-Saharan Africa were sleeping under a bed net. ${ }^{4}$ Insecticide-treated nets were credited with a $50 \%$ reduction in mortality across Africa from 2000 through $2015 .{ }^{6}$ Despite this progress, 2018 still saw 405,000 malaria deaths, with $94 \%$ occurring in the WHO Africa region. ${ }^{7}$ The WHO reported that Nigeria alone experienced $25 \%$ of global malaria cases and $24 \%$ of global malaria deaths in $2018 .{ }^{4}$ However, pyrethroids have long been the only insecticide recommended for bed nets by the $\mathrm{WHO}{ }^{8}$

Unfortunately, insecticide resistance threatens both maintaining existing successes and addressing the remaining burden. To gather data and help countries and donors respond, the WHO established its global plan for insecticide resistance management and its framework for a national plan for monitoring and management of insecticide resistance in malaria vectors. ${ }^{4}$ As of 2018, of the countries reporting insecticide monitoring with the WHO, $87.5 \%$ reported resistance to pyrethroids. Among the WHO regions, the Africa region had the highest share of sites with confirmed resistance. ${ }^{4}$ Over recent years, experts concluded, "The distribution and strength of this resistance has increased dramatically in recent years and now threatens the success

* Address correspondence to Donald S. Shepard, The Heller School for Social Policy and Management, Brandeis University, P.O. Box 549110, Waltham, MA 02454-9110. E-mail: shepard@brandeis.edu of control programs."9 They noted that resistance had "... increased mosquito survival, which is a prelude to rising incidence of malaria and fatalities, " 10 and ". . .could threaten the fragile gains that have been made in reducing malaria across Africa."11

Public health experts have also highlighted the urgency for adaptation and new tools at affordable costs. ${ }^{12}$ The WHO stated that using measured and specific application of insecticides can improve their long-term efficacy and decelerate the rate of development of vector resistance. ${ }^{13}$ However, new technologies and tools are needed to maintain malaria control in the face of increasing insecticide resistance. One of the most viable current solutions is to consider pyrethroidpiperonyl butoxide (PBO) long-lasting ITNs (LLINs) (PBO nets), which incorporate a synergist, $\mathrm{PBO}$, into the net with a pyrethroid insecticide. Piperonyl butoxide blocks the metabolic oxidase enzyme mechanism of the mosquito to enhance the effect of the pyrethroid (in the case of the product used in this study, deltamethrin).

Several laboratory studies have demonstrated the increased efficacy of PBO nets in bioassay tests in areas where conventional (pyrethroid-only) LLINs no longer effectively kill wild, resistant Anopheles mosquito populations. ${ }^{14,15}$ Two entomological studies in Nigeria showed the efficacy of PBO nets in areas of insecticide resistance. ${ }^{16,17}$ Advantages of the PBO net over the conventional LLINs on entomological indicators (e.g., mosquito mortality, mosquito density, and proportion of blood-fed mosquitoes) ranged from $17 \%$ to $63 \%$. $^{18}$ An entomological model predicted that PBO nets would reduce clinical malaria cases substantially ( 0.5 clinical cases per person per year) in areas of pyrethroid resistance in Africa. ${ }^{19}$

More recent findings underscored the value of PBO. In 2017, the WHO Pesticide Evaluation Scheme gave an interim recommendation to five bed nets with $\mathrm{PBO}$, which was subsequently converted to a WHO prequalification listing: PermaNet $^{\circledR} 3.0$ by Vestergaard (Lausanne, Switzerland), Olyset ${ }^{\circledR}$ Plus by Sumitomo Chemical (Tokyo, Japan), Veeralin by VKA 
Polymers (Tamil Nadu, India), and DawaPlus ${ }^{\circledR} 3.0$ and 4.0 by TANA Netting (Bangkok, Thailand). ${ }^{20-23}$ The WHO committees have noted the value of two PBO nets (PermaNet ${ }^{\circledR} 3.0^{24,25}$ and Olyset ${ }^{\circledR}$ Plus), leading the WHO to establish a new class of vector control products. ${ }^{26}$ In 2017 , the WHO issued a conditional recommendation for $\mathrm{PBO}$ nets to become a new class of vector control products for use in areas of pyrethroid resistance. ${ }^{27}$ The 2019 World Malaria Report recommended that national malaria programs and their partners consider the deployment of pyrethroid-PBO nets in areas with confirmed pyrethroid resistance and satisfying two other conditions. ${ }^{4}$ The need for such products applies to many endemic areas of Africa. ${ }^{28}$

However, PBO nets, like other newer products, tend to be more expensive than their traditional predecessors because of higher production costs, smaller volumes, and developers' need to recoup research and development costs. If procurement budgets were fixed, more expensive bed nets could lead to lower coverage of ITNs and/or less frequent replacement. Mathematical modelers ${ }^{19}$ and public health experts have thus called for cost-effectiveness studies. In response to this need, we examine the cost-effectiveness of PBO nets compared with conventional LLINs. Our study is based on 50 months of observed malaria case data (18 preceding and 32 following net distribution) corresponding to the entomological field study by Awolola et al. ${ }^{17}$ Our results are expressed in 2019 U.S. dollars. Whereas previous trials examined infections, which are sometimes asymptomatic and therefore do not entail any costs, this study focuses specifically on symptomatic malaria where the patient felt sufficiently ill to seek treatment and examines cost-effectiveness.

\section{METHODS}

Study setting. During the study period of 2010-2014, Nigeria reported resistance to all four classes of antimalarial insecticides monitored by the WHO (pyrethroid, DDT, carbamate, and organophosphate). ${ }^{29}$ Awolola et al. ${ }^{17}$ compared the entomological impact of PBO nets (PermaNet ${ }^{\circledR}$ 3.0) with conventional LLINs (PermaNet ${ }^{\circledR} 2.0$ ) in Remo North local government area of Ogun State, southwestern Nigeria. Their study selected three villages (Irolu, ljesha, and Ilara) some 3-5 km apart and matched on baseline insecticide resistance.

The study area is a forest zone with a rainy season extending from April to October (mean temperature $24^{\circ} \mathrm{C}$ ) and dry season from November to March (mean temperature $30^{\circ} \mathrm{C}$ ). The mean annual rainfall is $2,000 \mathrm{~mm}$, and the mean relative humidity is $78 \%$. The wider area consists of 15 agrarian communities of approximately 5,000 people each. Each community is surrounded by small cocoa and palm tree plantations, as well as small vegetable gardens. Herds of cattle and goats are commonly kept by nomadic herdsmen. Most (60-65\%) houses are constructed with brick walls and corrugated iron roofs, and the remaining houses generally have mud walls and thatched roofs (20-25\%). The ethnic group is primarily Yoruba with similar culture and traditions across the communities. Malaria is endemic with perennial transmission associated with Anopheles gambiae s.s.

Interventions. In March 2012, under an entomological study, the villages were randomly assigned to the study arms to receive one of three types of bed nets. Residents in Irolu received PBO nets (PermaNet ${ }^{\circledR} 3.0$ ), those in ljesha received conventional (pyrethroid-only) LLINs (PermaNet ${ }^{\circledR} 2.0$ ), and those in llara received untreated polyester nets. The nets were distributed free of charge to community residents to cover all sleeping spaces. Irolu, with 550 residents, received 137 nets, or 0.249 per person. ljesha, with 590 residents, received 147 nets, also 0.249 per person. During the follow-up, the proportion of blood-fed mosquitoes in Irolu (with PBO nets) was only $7.3 \%(95 \% \mathrm{Cl}$ : $2.8-8.1 \%)$ compared with $22.2 \%(\mathrm{Cl}$ : 18.4-26.5\%) in ljesha (with conventional LLINs) and $56.9 \%$ (Cl: $51.2-62.8 \%$ ) in llara (with untreated nets). ${ }^{17}$ In all villages, participants experiencing episodes of febrile illness were encouraged to visit the local health center and were tested for malaria using a rapid diagnostic test as part of their clinical management. All villages were served by the same health center, which treated residents of all three villages equally and maintained similar practices over the entire study period.

Epidemiological and statistical analyses of cases averted. After the completion of the entomological study, the original entomological investigators decided to analyze the existing clinical data from Irolo and ljesha residents for a pragmatic trial. There was no contact with any residents and no new original data obtained. The original investigators tabulated the clinic's existing anonymized malaria test results, where each test was classified as confirmed positive or negative for malaria, or unconfirmed (i.e., indeterminate). Test results were summarized by month, village of residence, and gender for 50 months from September 2010 through October 2014.

To avoid underestimating clinical malaria, we allocated the unconfirmed cases according to gender-specific proportions for tests with definitive outcomes (i.e., $31.1 \%$ in male patients and $29.8 \%$ in female patients). Combining the definitive test outcomes and probabilistically attributed outcomes gave total estimated positives (malaria cases) by village and month.

Our analytical data set had observations by village by month with the estimated number of malaria cases (CASES) as the dependent variable and three dichotomous independent variables. The first independent variable, IROLU, was scored 1 for Irolu, the village which subsequently got PBO nets (PermaNet ${ }^{\circledR} 3.0$ ), and 0 for ljesha, the village which subsequently received conventional LLINs (PermaNet ${ }^{\circledR}$ 2.0). The second independent variable, NETS, indicating that one of the two types of ITNs had been distributed in the village, was scored 1 if the time period was March 2012 or later, indicating that nets had been distributed, and 0 otherwise. The third independent variable, INTERACTION, representing the interaction between IROLU and NETS indicated that PBO nets had been distributed as contrasted with conventional LLINs. Our statistical analysis used negative binomial regression on CASES with IROLU, NETS, and INTERACTION as independent variables and 100 observations covering 50 months in each village. The coefficient for INTERACTION provided our best estimate of the incremental benefit of the PBO net over the conventional LLINs. This statistical model controlled for any differences in exposure, mosquito density, and population between village and changes in temperature, humidity, rainfall, and pyrethroid resistance over time.

Impact on disability-adjusted life years (DALYs). We converted the change in incidence to DALYs averted from mortality based on 2014 global numbers of deaths and case rates reported by the $\mathrm{WHO} .{ }^{3}$ As malaria deaths occur primarily in young children and in low- and middle-income countries, we approximated health loss from each malarial death by the healthy life expectancy at birth. ${ }^{30}$ Discounting life years lost at 
the recommended rate of $3 \%$ per year, ${ }^{31,32}$ we calculated that the death of a person from malaria represents a loss of about 27.85 discounted DALYs. When prorated overall cases (including the nonfatal ones) globally, this loss became 0.0495 discounted health-adjusted life years lost from mortality per case. $^{29,30,32}$ The overall DALY burden per malaria case was first calculated in undiscounted terms, as reported by recent Global Burden of Disease studies. ${ }^{33}$ It was then adjusted for discounting based on the difference between the mortality burden in discounted and undiscounted terms.

Intervention costs. Our framework for cost analysis, built on cost analysis of other malaria vector control programs in sub-Saharan Africa, entailed quantifying, valuing, and summing the ingredients (inputs) in each arm. ${ }^{34}$ Unit procurement costs of conventional LLINs and PBO nets, excluding their distribution, were based on average of online customized reference prices from the Global Fund from February 10, 2020, presumably reflecting costs in $2019 .{ }^{35}$ A 2012 costing model suggested the least expensive delivery cost (through fixed clinics) averaged US $\$ 1.40 .{ }^{36}$ For the present study, the most relevant cost is the incremental cost of a net addressing resistance over a conventional net. As the size and weight for the conventional LLIN and PBO net are approximately similar, distribution costs would be virtually identical. Therefore, the incremental distribution cost would be nil.

We estimated bed nets lost in the distribution process through data by computing the ratio of nets distributed in Nigeria compared with the number of nets delivered to the country. ${ }^{37}$ We analyzed data from the study year (2012) through 2015. In the 4 years, the number of nets delivered (98.6 million) was 1.094 times the number distributed ( 90.1 million). We adjusted the ratio upward to 1.10 to factor in nets lost, damaged, or repurposed after distribution to households. ${ }^{36}$

Cost-effectiveness analysis. To conduct our cost-effectiveness analyses, we created a deterministic Excel model (Microsoft Office Professional Plus 2013, Redmond, WA) to estimate health impacts and costs in each of these subsystems. Bed nets consume resources in the prevention subsystem but, if effective, generate savings to the treatment subsystem through expenses on medical services averted.

Our model calculates cost-effectiveness from two perspectives reflecting the organizational structure within the health system: a prevention perspective and an overall "health systems perspective." The prevention or public health perspective counts only the resources used aimed at avoiding illness, whereas the overall health systems perspective also includes the savings from malaria treatment averted by the preventive activities.

To interpret the cost-effectiveness ratios, we noted that countries' limited prevention budget must cover net purchases, indoor residual spraying (IRS), and other malaria prevention products. The Commission on Macroeconomics and Health recommended that incremental cost-effectiveness ratios (ICERs) be compared with the target country's per capita gross domestic product (GDP). ${ }^{38}$ Interventions with an ICER at or below one time per capita GDP were considered highly costeffective, an important consideration in subsequent guidelines. $^{39}$ The 2019 per capita gross national income (GNI) of Nigeria, which is similar to its per capita GDP, was US $\$ 2,030.40$

Sensitivity analysis. The central value for each parameter is our most likely value. The $\mathrm{Cl}$ around the coefficient INTERACTION formed the basis for our sensitivity analysis. The value of smallest magnitude (least reduction in $95 \% \mathrm{Cl}$ ) served as the worst case for the sensitivity analysis, whereas the largest magnitude (greatest reduction) served as the best case for the sensitivity analysis.

\section{RESULTS}

Effectiveness. Over the observation period, 2,738 febrile cases were recorded in both villages combined. The patients' average age was 24.8 years, with a range of $1-87$ years. The gender mix was fairly even (52\% male and $48 \%$ female). The village with the PBO net showed significantly fewer malaria cases than the village with the conventional LLIN. Table 1 shows the differences in test outcomes in febrile illnesses between PBO nets compared with conventional LLINs using arithmetic and geometric means of case numbers. Comparing the ratios of before and after numbers of cases shows reductions of $32 \%$ based on the arithmetic mean and $31 \%$ based on the geometric mean.

Table 2 shows the results of the negative binomial regression. The NETS coefficient shows that conventional nets reduced monthly malaria cases by $34.7 \%$ compared with the pre-period (no nets) (i.e., 1-exp(-0.427). The key coefficient (INTERACTION), corresponding to the interaction term, is -0.406 and highly statistically significant $(P<0.01)$. This indicates that $\mathrm{PBO}$ nets reduced monthly malaria cases by $33.4 \%$ compared with conventional LLINs (i.e., 1-exp(-0.406)), with a $95 \% \mathrm{Cl}$ of $10.2-50.6 \%$. Both estimates are similar to the estimates from the comparisons of ratios.

Figure 1 displays numbers of malaria cases by month for the two villages. The vertical dotted line at month 19 demarcates March 2012, when the nets were installed in the two villages. The solid (orange) line shows the numbers for Irolu (which received the $\mathrm{PBO}$ nets), and the dashed (blue) line shows the numbers for ljesha (which received the conventional LLIN). The fluctuating lines to the left of the installation show the monthly numbers of cases before net installation in each village, and the horizontal (red) line shows that average number of cases per month before net installation (10.61). The upper (gray) horizontal line after installation is the average value estimated from the regression equation if Irolu had received conventional nets (6.93), and the lower (yellow) horizontal line

TABLE 1

Aggregate monthly number of malaria cases by village and time period

\begin{tabular}{|c|c|c|c|c|c|}
\hline Village and treatment & Period (nets) & Arithmetic mean & SE & Geometric mean & Relative error \\
\hline ljesha (conventional LLINs) & Pre (no nets) & 9.19 & 0.74 & 8.76 & $7.4 \%$ \\
\hline Irolu (PBO nets) & Pre (no nets) & 10.61 & 0.33 & 10.53 & $3.0 \%$ \\
\hline ljesha (conventional LLINs) & Post (conventional LLINs) & 5.91 & 0.49 & 5.19 & $11.1 \%$ \\
\hline Irolu (PBO nets) & Post (PBO nets) & 4.67 & 0.60 & 4.32 & $9.3 \%$ \\
\hline
\end{tabular}

LLINs = long-lasting insecticide treated nets; PBO = piperonyl butoxide; SE = standard error. ljesha and Irolu had 550 and 590 residents, respectively 
TABLE 2

Negative binomial regression on the number of statistically positive malaria cases by arm by month (natural log scale)

\begin{tabular}{lrrrrrr}
\hline & \multicolumn{2}{c}{ Regression estimates } & & \multicolumn{3}{c}{ Relative impact on cases } \\
\cline { 2 - 3 } \cline { 6 - 7 } \multicolumn{1}{c}{ Variable } & Coefficient & Significance & & Estimate & \multicolumn{1}{c}{ Lower } & \multicolumn{1}{c}{ Upper } \\
\hline IROLU & 0.144 & 0.176 & & $15.5 \%$ & $-6.2 \%$ & $42.2 \%$ \\
NETS & -0.427 & 0.000 & & $-34.7 \%$ & $-47.0 \%$ & $-19.6 \%$ \\
INTERACTION & -0.406 & 0.008 & & $-33.4 \%$ & $-50.6 \%$ & $-10.2 \%$ \\
Constant & 2.218 & 0.000 & & 8.192 & 6.893 & 9.705
\end{tabular}

LLINs = long-lasting insecticide treated nets. IROLU measures the difference in the natural log scale of monthly incidence by which the baseline average of Irolu exceeds that of ljesha; NETS (any bed nets installed) measures the effect of LLINs installation in ljesha compared with its pre-net value; INTERACTION denotes the interaction term, showing the net difference of piperonyl butoxide on cases compared with conventional LLINs; Estimate shows the best estimate; lower and upper are the corresponding $95 \%$ confidence bounds.

after installation is the estimated value from the regression in Irolo after receiving the PBO nets (4.62). The distance between these two horizontal lines (2.31, calculated as 6.93-4.62) corresponds to the incremental impact (estimated monthly number of clinical malaria cases averted by PBO nets over conventional LLINs).

Illness and treatment costs averted. To assess illness averted, we needed to calculate the health gain from each malaria case averted. To do this, we relied on data from Murray et al., ${ }^{30}$ as shown in Table 3.

To determine treatment costs averted, we needed to calculate the cost of treating a malaria case. Here, we relied on a 2013 study of the costs of ambulatory and hospitalized malaria episodes in Nigeria ${ }^{42}$ adapted from global studies. ${ }^{43}$ This Nigerian study, obtaining costs at a secondary public hospital, a mission hospital, and three outpatient clinics, found that the health system (i.e., provider) cost per case averaged $\$ 30.42$ for an outpatient case and $\$ 48.02$ for an inpatient case.
As healthcare spending tends to vary across and within countries with per capita GNI, this indicator provided a reasonable proxy for the resources available to the country's health system. We therefore adjusted the 2013 cost per episode to 2019 levels in proportion to the 2013-2019 change in per capita GNI. We calculated this factor (0.7463) from World Bank data. ${ }^{40}$ As we are not aware of quantitative estimates from Nigeria on the proportion of malaria cases treated in the formal health system that were hospitalized, we used the share $(1.7 \%)$ from a rigorous study in another sub-Saharan African country as a proxy. ${ }^{44}$ Using this share as a weight, we calculated the average cost of a treated case as $\$ 22.93$.

Cost-effectiveness results. Table 4 presents the costeffectiveness and cost-benefit analyses. The cost-effectiveness analysis is presented first from a prevention perspective that considers public sector outlays on prevention. It does not include any expected savings in reduced treatment costs averted. The rationale for this perspective is that such savings are distributed among all health facilities in the country, both public and private, and cannot readily be captured and used to offset prevention resources. The second analysis, from a health systems perspective, includes the offsets from treatment costs averted. Under this perspective, even if curative care budgets cannot be directly reduced to fund prevention, these resources could still be redirected by households or health facilities. They could support treatment for Nigeria's other health problems, often affecting the same agegroups. The overall health systems perspective is the one conventionally recommended in cost-effectiveness analysis. ${ }^{32}$ Under this perspective, predicted savings in the cost of malaria treatment offset some of the cost of the more expensive bed nets.

The concurrent (2013) Nigeria Demographic and Health Survey ${ }^{45}$ found that $32.7 \%$ of children younger than 5 years with a febrile illness received an appropriate antimalarial drug for that illness. This

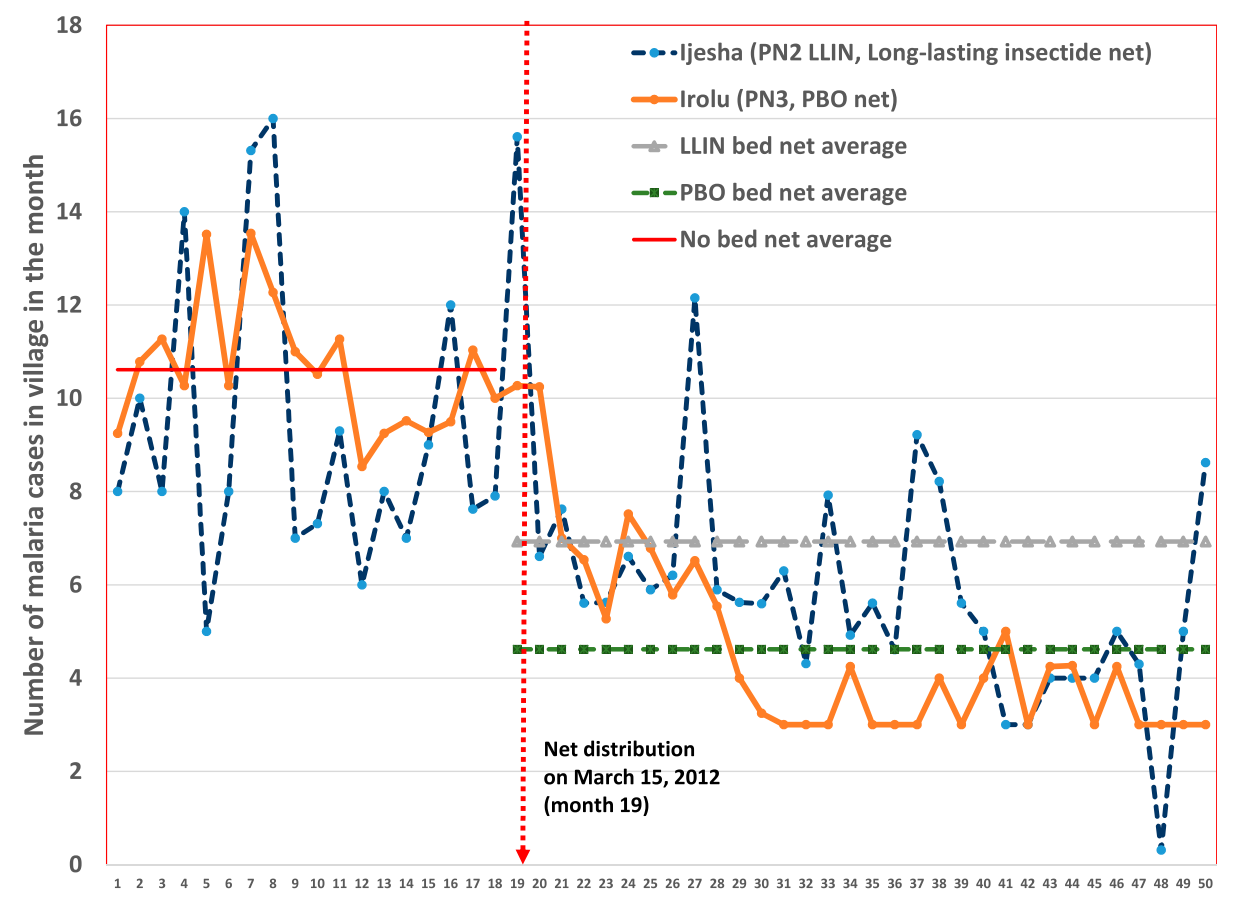

Months (1 is Sept 2010)

FIGURE 1. Number of malaria cases by village by month. $\mathrm{PBO}=$ piperonyl butoxide; $\mathrm{PN} 2=$ PermaNet 2.0 (conventional Long-lasting insectide net [LLIN]); PN3 = PermaNet 3.0 (PBO net). This figure appears in color at www.ajtmh.org. 
TABLE 3

Derivation of discounted DALYs lost due to mortality per malaria case, 2013

\begin{tabular}{|c|c|c|}
\hline Item & Value & Formula or source \\
\hline Annual global malaria cases (millions) & 228 & $\mathrm{WHO}^{4}$ \\
\hline Annual global malaria deaths (millions) & 0.405 & $\mathrm{WHO}^{4}$ \\
\hline Malaria case fatality rate & 0.0018 & $(2) /(3)$ \\
\hline Discount rate per year & $3 \%$ & Neumann ${ }^{32}$ \\
\hline \multicolumn{3}{|l|}{ HALE at birth in developing } \\
\hline Male (undiscounted) & 59.47 & $\begin{array}{l}\text { Murray }^{30}, \\
\text { p2165 }\end{array}$ \\
\hline Female (undiscounted) & 62.65 & $\begin{array}{c}\text { Murray }^{30} \text {, } \\
\text { p2165 }\end{array}$ \\
\hline Overall (undisco & 61.06 & {$[(10)+(11)] / 2$} \\
\hline Overall (discount & 27.85 & $\begin{array}{l}\text { Present value } \\
\quad \text { using (4) \& (11) }\end{array}$ \\
\hline \multicolumn{3}{|l|}{ Loss per malaria case in HALE } \\
\hline Undiscounted & 0.1085 & $(3) \times(8)$ \\
\hline Discounted & 0.0495 & (3) $\times(7)$ \\
\hline Discounting adjustment (difference) & 0.0590 & (9)-(10) \\
\hline Global DALY burden (millions & 45.000 & $\mathrm{GBD}^{41}, \mathrm{p} 1789$ \\
\hline \multicolumn{3}{|l|}{ Global DALY burden per case } \\
\hline Undiscounted & 0.1974 & $(12) /(1)$ \\
\hline Discounted (adjusted) & 0.1384 & (13)-(11) \\
\hline
\end{tabular}

percentage did not vary systematically by age when examined by single years of age. We therefore assumed that the same percentage would apply to all age-groups. Thus, under the health systems perspective, for every case of malaria averted, 0.327 treated cases and their associated costs would be averted. Adjusting for differences in the phasing of the question compared with the previous survey, the latest (2018) Nigeria Demographic and Health Survey gave comparable findings. ${ }^{46}$

Under the prevention perspective, the ICER was $\$ 11$ per DALY averted, with a $95 \% \mathrm{Cl}$ of $\$ 8-\$ 37$. As all of these values are substantially below Nigeria's projected 2019 per capita GNI of US $\$ 2,030 .^{40}$ The PBO was highly cost-effective under the prevention perspective. Under the health systems perspective, the cost-effectiveness ratios showed cost savings for all cases. The central value is $-\$ 43$, with a $95 \% \mathrm{Cl}$ of $-\$ 47$ to $-\$ 17$. This means that using a PBO net in place of a conventional LLIN improves health and saves healthcare resources, even under the least favorable estimate of efficacy.

The benefit/cost ratio (based on the health systems perspective) was 201. These results mean that US \$1 invested in improved bed nets generates US \$201 in the economic benefits (with a range of $\$ 61-\$ 304$ ), or one to two orders of magnitude over the added costs-very highly favorable.

\section{DISCUSSION}

Because the PBO and conventional nets were deployed and compared by village, indirect benefits accrued to all residents of the PBO village because of fewer infected mosquitoes. These benefits extended to residents temporarily outside their net, those not using a bed net, and other neighbors, that is, community benefit. Under the prevention perspective often used by donors and health ministries, in which the savings in curative care are not incorporated, PBO nets would be highly cost-effective with the incidence and incremental effectiveness observed in Nigeria. Our effectiveness results from clinical cases are consistent with previously noted entomological data of a significantly lower rate of blood-fed mosquitoes with PBO nets than conventional LLINs. ${ }^{17}$

All the benefit/cost ratios are well above the critical threshold of 1.0, indicating that the economic value of the health gains substantially exceeds the cost of generating them. The results are highly favorable because the added benefits in areas of pyrethroid resistance are substantial, whereas the added costs are minimal (no added distribution costs for PBO nets and only small additional procurement costs).

We made the assumption that the PBO net would have the same 3-year lifetime as conventional LLINs. As the PBO net used in this study (PermaNet 3.0) has a reinforced border, it could have greater resistance to damage, another attribute noted by the WHO. ${ }^{18}$ If so, its longer duration and greater protection against malaria infections could be more favorable than estimated here.

The ICERs of $\$ 11$ (95\% Cl: \$8-\$37) under the prevention perspective and $-\$ 43(95 \% \mathrm{Cl}$ : $-\$ 47$ to $-\$ 17)$, indicating costsaving, under the health systems perspective are excellent. These are even more favorable than another highly valued technology, IRS, under a health systems perspective of $\$ 18$ in a modeling study ${ }^{47}$ and $\$ 139$ in an empirical study in Tanzania. ${ }^{34}$ Our results suggest that other innovative nets to address pyrethroid resistance may also be highly cost-effective. For example, research from Burkina Faso found promising results from placing a piece of a more potent insecticidetreated netting attached perpendicularly to the top of a traditional bed net. ${ }^{48}$

Four limitations must be acknowledged. First, the data on effectiveness were obtained from just one pair of villages using nets from a single manufacturer and were compiled retrospectively from clinic registers after the completion of the entomological study. However, the availability of a relatively long follow-up (32 months after net installation), a consistent source of data for both villages (the same clinic), a common manufacturer; the application of 18 months of pre-net data using an interaction model; and the use of a laboratory test conducted by disinterested clinic staff all reduce the risk of confounding or spurious findings. Second, the epidemiologic data were obtained several years ago. However, the growth of insecticide resistance would mean that if the study were replicated now, the results would likely be even more favorable. Third, as PBO nets are a relatively new technology, their prices are likely to continue to fall with competition and economies of scale, as occurred with conventional LLINs. Indeed, one manufacturer's 2017 price per PBO net $(\$ 4.44)$ was $3.5 \%$ below its 2015 value $(\$ 4.60$, Helen Pates Jamet, then with Vestergaard, personal communication, August 4, 2017). Assuming the incremental cost of a PBO net over a conventional LLIN remains the same or shrinks in the future, the economic advantage of PBO nets will remain at least as favorable. Fourth, the cost per malaria case treated is based on just one Nigerian study with weights from another country. However, even under the prevention perspective, which does not consider cost offsets, the results are highly favorable.

\section{CONCLUSION}

A 2015 WHO Evidence Review Group noted the need for more evidence on $\mathrm{PBO}$ nets. ${ }^{49}$ A follow-up from a cluster randomized trial of a PBO net (Olyset Plus by Sumitomo) from Tanzania's Lake Zone ${ }^{50}$ revealed that subjects who received 
TABLE 4

Cost-effectiveness and cost-benefit analyses*

\begin{tabular}{|c|c|c|c|c|}
\hline Variable & Central & Worst & Best & Source \\
\hline \multicolumn{5}{|l|}{ Effectiveness } \\
\hline (1) Clinical malaria cases per person per year, conventional LLIN & 0.1511 & 0.1511 & 0.1511 & Observed in trial \\
\hline (2) Percentage (relative) benefit of PBO net over conventional LLIN & $33.4 \%$ & $10.2 \%$ & $50.6 \%$ & Table 2 \\
\hline $\begin{array}{l}\text { (3) Reduction in malaria cases from PBO nets versus conventional } \\
\text { LLINs }\end{array}$ & 0.050 & 0.015 & 0.076 & $(1) \times(2)$ \\
\hline (4) Persons per PBO net & 4.0 & 4.0 & 4.0 & Observed in trial \\
\hline (5) Incremental cases averted per PBO net per year & 0.2019 & 0.0616 & 0.3058 & $(3) \times(4)$ \\
\hline (6) Lifetime of PBO net (years) & 3.0 & 3.0 & 3.0 & Expert opinion \\
\hline (7) Incremental PV number of cases averted per net over its lifetime & 0.5710 & 0.1744 & 0.8650 & PV using (6) \& (7) \\
\hline (8) Incremental discounted DALYs averted per case averted & 0.1384 & 0.1384 & 0.1384 & Table 3 \\
\hline (9) Incremental discounted DALYs averted per PBO net over its lifetime & 0.0790 & 0.0241 & 0.1197 & $(7) \times(8)$ \\
\hline \multicolumn{5}{|l|}{ Cost offsets } \\
\hline (10) Nigeria cost of one treated case & $\$ 22.93$ & $\$ 22.93$ & $\$ 22.93$ & Onwujekwe ${ }^{42}$ \\
\hline (11) \% Of cases treated & $32.7 \%$ & $32.7 \%$ & $32.7 \%$ & $\mathrm{NPC}^{45}$ \\
\hline (12) Average treatment cost per case (including untreated) & $\$ 7.50$ & $\$ 7.50$ & $\$ 7.50$ & $(10) \times(11)$ \\
\hline (13) Average treatment cost averted per PBO net over its lifetime & $\$ 4.28$ & $\$ 1.31$ & $\$ 6.49$ & $(7) \times(12)$ \\
\hline \multicolumn{5}{|l|}{ Intervention costs } \\
\hline (14) Cost per PBO net distributed & $\$ 3.18$ & $\$ 3.18$ & $\$ 3.18$ & Global Fund ${ }^{35}$ \\
\hline (15) Cost per conventional LLIN distributed & $\$ 2.36$ & $\$ 2.36$ & $\$ 2.36$ & Global Fund ${ }^{35}$ \\
\hline $\begin{array}{l}\text { (16) Incremental cost of PBO net over conventional LLIN per net } \\
\text { distributed }\end{array}$ & $\$ 0.82$ & $\$ 0.82$ & $\$ 0.82$ & $(14)-(15)$ \\
\hline (17) Loss factor: Nets distributed/nets delivered & 1.10 & $\$ 1.10$ & $\$ 1.10$ & $\mathrm{AMP}^{37}$ \\
\hline \multicolumn{5}{|l|}{ Incremental cost of PBO nets over conventional LLINs per net delivered } \\
\hline (18) Prevention perspective & $\$ 0.90$ & $\$ 0.90$ & $\$ 0.90$ & $(16) \times(17)$ \\
\hline (19) Health systems perspective & $-\$ 3.38$ & $-\$ 0.41$ & $-\$ 5.58$ & $(18) \times(13)$ \\
\hline \multicolumn{5}{|l|}{ ICER, \$/DALY } \\
\hline (20) Based on prevention perspective & $\$ 11$ & $\$ 37$ & $\$ 8$ & $(18) /(9)$ \\
\hline (21) Per capita GNI of Nigeria, 2019 & $\$ 2,030$ & $\$ 2,030$ & $\$ 2,030$ & World Bank ${ }^{40}$ \\
\hline (22) As fraction of GNI per capita (lower is better) & 0.006 & 0.018 & 0.004 & $(20) /(21)$ \\
\hline (23) Based on health systems perspective & $-\$ 43$ & $-\$ 17$ & $-\$ 47$ & $(19) /(9)$ \\
\hline (24) Interpretation (negative is cost saving, CS) & CS & CS & CS & Row (23) \\
\hline \multicolumn{5}{|l|}{ Benefit-cost analysis } \\
\hline (25) Economic value of DALYs gained and cost offsets per net & $\$ 164.70$ & $\$ 50.30$ & $\$ 249.51$ & $(9) \times(21)+(13)$ \\
\hline (26) Benefit/cost ratio & 201 & 61 & 304 & $(25) /(16)$ \\
\hline
\end{tabular}

${ }^{*} \mathrm{AMP}=$ Alliance for Malaria Prevention; CS = cost-saving; DALY = disability-adjusted life year; GNI = gross national income; ICER = incremental cost-effectiveness ratio; LLINs = long-lasting insecticide net; NPC = National Population Commission (Nigeria); PBO = piperonyl butoxide; PV = present value; central denotes the most likely estimate for the parameter; worst and best indicate the possible range of the parameter based on trial outcomes, leading to the least and most favorable cost-effectiveness and benefit/cost ratios, respectively. Currency is in $2019 \mathrm{U}$.S. dollars.

these nets experienced fewer malarial infections than those who received conventional LLINs. ${ }^{28}$ From these results, the WHO issued a conditional recommendation for pyrethroidPBO nets to be designated as a new class of vector control products and should be deployed in areas where the main malaria vector(s) have pyrethroid resistance. ${ }^{27}$ This endorsement would include many endemic areas in Africa where conventional LLINs are currently used. ${ }^{28} \mathrm{~A}$ pragmatic cluster randomized trial from Uganda found significantly lower parasitemia prevalence in areas with PBO nets than conventional LLINs (e.g., prevalence ratio $0.84, P<0.05$ at 18 months). ${ }^{51}$

Our results reinforce and extend results from the Tanzanian $^{52}$ and Ugandan ${ }^{51}$ trials and previous modeling work $^{19,53-55}$ in several ways. It includes epidemiological and illness data, rather than just entomological or infection data; examines another part of Africa; includes over 4 years of malaria data; and incorporates economic analysis. The pragmatic design indicates that many preventive programs may provide opportunities for analysis of existing clinical data for epidemiologic and cost-effectiveness analyses. Although this pragmatic design requires at least 1 year of pre-intervention data, it avoids the expense of delineating multiple clusters and regularly testing samples of residents from each cluster for infection. We showed that in an area with vectors with high pyrethroid resistance, PBO nets averted substantially more malaria cases than conventional LLINs, were highly cost-effective from a prevention perspective, and were cost-saving from a health systems perspective when factoring in treatment costs averted.

Received August 5, 2020. Accepted for publication October 26, 2020.

Published online December 21, 2020.

Acknowledgments: We thank Melinda Hadi (Vestergaard) and Helen Pates Jamet (Bill \& Melinda Gates Foundation) for constructive comments and Clare Hurley (Brandeis University) for editorial assistance.

Financial support: This study was supported in part by a grant (OPP 1038575) from the Bill \& Melinda Gates Foundation to Brandeis University.

Disclosure: D. S. S. has received grant support from Takeda Vaccines, Inc., Abbott, Inc., and Sanofi Pasteur unrelated to this study.

Authors' addresses: Donald S. Shepard and John U. Odumah, Heller School for Social Policy and Management, Brandeis University, Waltham, MA, E-mails: shepard@brandeis.edu, and jodum@brandeis.edu. Samson T. Awolola, Molecular Entomology and Vector Control Research Laboratory, Nigerian Institute of Medical Research, Yaba Lagos, Nigeria, E-mail: awololas@hotmail.com.

This is an open-access article distributed under the terms of the Creative Commons Attribution (CC-BY) License, which permits unrestricted use, distribution, and reproduction in any medium, provided the original author and source are credited.

\section{REFERENCES}

1. United Nations, 2015. Millenium Development Goals and Beyond 2015-Goal 6 Combat HIVIAIDS Malaria and Other Diseases. 
Available at: https://www.un.org/millenniumgoals/aids.shtml. Accessed December 7, 2020.

2. United Nations, 2015. Sustainable Development Goals-17 Goals to Transform Our World. Available at: https://www.un.org/ sustainabledevelopment/. Accessed December 7, 2020.

3. World Health Organization, 2015. Global Technical Strategy and Targets for Malaria 2016-2030 (WHA68.2). Available at: https:// www.who.int/malaria/areas/global_technical_strategy/en/. Accessed March 30, 2020.

4. World Health Organization, 2019. World Malaria Report 2019. Available at: https://www.who.int/news-room/feature-stories/ detail/world-malaria-report-2019. Accessed March 30, 2020.

5. CDC, 2019. Malaria: Insecticide Treated Nets (ITNs). Available at: https://www.cdc.gov/malaria/malaria_worldwide/reduction/ itn.html. Accessed March 30, 2020.

6. Bhatt $S$ et al., 2015. The effect of malaria control on Plasmodium falciparum in Africa between 2000 and 2015. Nature 526: 207-211.

7. World Health Organization, 2020. Malaria: Key Facts. Available at: https://www.who.int/news-room/fact-sheets/detail/malaria. Accessed March 30, 2020.

8. Najara J, Zaim M, 2003. Malaria Vector Control: Decision Making Criteria and Procedures for Judicious Use of Insecticides. Geneva, Switzerland: World Health Organization.

9. Ranson H, Lissenden N, 2016. Insecticide resistance in African Anopheles mosquitoes. A worsening situation that needs urgent action to maintain malaria control. Trends Parasitol 32: 187-196.

10. Hemingway $J$ et al., 2016. Averting a malaria disaster: will insecticide resistance derail malaria control? Lancet 387: 1785-1788.

11. Edi C, Koudou B, Jones C, Weetman D, Ranson H, 2012. Multipleinsecticide resistance in Anopheles gambiae mosquitoes, Southern Cote d'Ivoire. Emerg Infect Dis 18: 1508-1511.

12. Hemingway J, Shretta R, Wells T, Bell D, Djimde A, Achee N, Qi G, 2016. Tools and strategies for malaria control and elimination: what do we need to achieve a grand convergence in malaria? PLoS Biol 14: e1002380.

13. World Health Organization, The Health and Environment Linkages Initiative, 2020. Malaria Control: the Power of Integrated Action. Available at: https://www.who.int/heli/risks/vectors/malariacontrol/ en/. Accessed March 24, 2020.

14. Abílio A, Marrune P, de Deus N, Mbofana F, Muianga P, Kampango A, 2015. Bio-efficacy of new long-lasting insecticide-treated bed nets against Anopheles funestus and Anopheles gambiae from central and northern Mozambique. Malar J 14: 352.

15. Yewhalaw D, Asale A, Tushune K, Getachew Y, Duchateau L, Speybroeck N, 2012. Bio-efficacy of selected long-lasting insecticidal nets against pyrethroid resistant Anopheles arabiensis from south-western Ethiopia. Parasit Vectors 5: 159.

16. Adeogun A, Olojede J, Oduola A, Awolola T, 2012. Village-scale evaluation of PermaNet 3.0: an enhanced efficacy combination long-lasting insecticidal net against resistant populations of Anopheles gambiae s.s. Malar Control Elimin 1: 235543.

17. Awolola S, Adeogun A, Olojede J, Oduola A, Oyewole I, Amajoh C, 2014. Impact of PermaNet ${ }^{\circledR} 3.0$ on entomological indices in an area of pyrethroid resistant Anopheles gambiae in southwestern Nigeria. Parasit Vectors 2: 236.

18. World Health Organization, 2015. Vector Control Policy Updates and Priorities (Vector Control Unit, Global Malaria Program). Geneva, Switzerland: WHO.

19. Churcher T, Lissenden N, Griffin J, Worrall E, Ranson H, 2016. The impact of pyrethroid resistance on the efficacy and effectiveness of bednets for malaria control in Africa. eLife 5: e16090.

20. World Health Organization, 2008. Report of the Eleventh WHOPES Working Group Meeting, 10-13 December 2007. Geneva, Switzerland: WHO.

21. World Health Organization, 2009. Report of the Twelfth WHOPES Working Group Meeting, 8-11 December 2008. Geneva, Switzerland: WHO.

22. World Health Organization, 2012. Report of the Fifteenth WHOPES Working Group Meeting, 8-22 June 2012. Geneva, Switzerland: WHO.

23. World Health Organization, 2016. Report of the Nineteenth WHOPES Working Group Meeting, 8-11 February 2016. Geneva, Switzerland: WHO.

24. Vestergaard, 2015. Technical Basis for Deployment of PermaNet巴 3.0 in Areas with Pyrethroid-Resistant Malaria vectors. Available at: https://pdfs.semanticscholar.org/035a/ 4b80e477121469a9a3a030e2efb0c1e85e13.pdf. Accessed March 30, 2020.

25. World Health Organization, 2017. Neglected Tropical Diseases, Vector Control Advisory Group (VCAG). Geneva, Switzerland: WHO.

26. World Health Organization, 2017. Global Malaria Programme: Conditions for Deployment of Mosquito Nets Treated with Pyrethroid and Piperonyl Butoxide. Recommendations. Geneva, Switzerland: World Health Organization. Available at: https:// apps.who.int/iris/bitstream/handle/10665/258939/WHO-HTMGMP-2017.17-eng.pdf. Accessed December 7, 2020.

27. World Health Organization, 2017. Conditions for Deployment of Mosquito Nets Treated with a Pyrethroid and Piperonyl Butoxide. Available at: https://www.who.int/malaria/publications/ atoz/use-of-pbo-treated-llins/en/. Accessed April 20, 2020.

28. Protopopoff $\mathrm{N}$ et al., 2018. Effectiveness of a long-lasting piperonyl butoxide-treated insecticidal net and indoor residual spray interventions, separately and together, against malaria transmitted by pyrethroid-resistant mosquitoes: a cluster, randomised controlled, two-by-two factorial design trial. Lancet 391: 1577-1588.

29. World Health Organization, 2017. World Malaria Report. Geneva, Switzerland: WHO.

30. Murray $C$ et al., 2015. Global, regional, and national disabilityadjusted life years (DALYs) for 306 diseases and injuries and healthy life expectancy (HALE) for 188 countries, 19902013 quantifying the epidemiological transition. Lancet 386 : 2145-2149.

31. Jamison D, Breman J, Measham A, Alleyne G, Claeson M, Evans D, Jha P, Mills A, Musgrove P, eds., 2006. Disease Control Priorities, 2nd edition. New York, NY: Oxford University Press.

32. Neumann P, Sanders G, Russell L, Siegel J, Ganiats T, 2017. Cost-Effectiveness Analysis in Health and Medicine, 2nd edition. New York, NY: Oxford University Press.

33. Global Burden of Disease Study 2013 Collaborators, 2015. Global, regional, and national incidence, prevalence, and years lived with disability for 301 acute and chronic diseases and injuries in 188 countries, 1990-2013: a systematic analysis for the Global Burden of Disease Study 2013. Lancet 386: 743-800.

34. Shepard DS et al., 2016. Cost-effectiveness of insecticide-treated wall liner and indoor residual spraying to prevent malaria in Kenya and Tanzania (Poster number 1665). 65th Annual Meeting of the American Society of Tropical Medicine \& Hygiene. Atlanta, GA: American Society of Tropical Medicine and Hygiene.

35. The Global Fund, 2020. Reference Price List. Geneva: the Global Fund for AIDS, Tuberculosis and Malaria. Available at: https:// www.theglobalfund.org/media/5861/psm_llinreferenceprices_ table_en.pdf. Accessed October 17, 2020.

36. Pulkki-Brannstrom AM, Wolff C, Brannstrom N, Skordis-Worrall J, 2012. Cost and cost effectiveness of long-lasting insecticidetreated bed nets-a model-based analysis. Cost Eff Resour Alloc 10: 5

37. Alliance for Malaria Prevention (AMP), 2017. AMP Conference Call Minutes. Available at: https://allianceformalariaprevention. com/conference-call-minutes/2017-conference-call-minutes/. Accessed December 7, 2020.

38. World Health Organization, 2001. Macroeconomics and Health: Investing in Health for Economic development. Report of the Commission on Macroeconomics and Health. Available at: https://apps.who.int/iris/handle/10665/42463. Accessed March 30, 2020.

39. Bertram M, Lauer J, De Joncheere K, Edejer T, Hutubessy R, Kieny MP, Hilla S, 2016. Cost-effectiveness thresholds: pros and cons. Bull World Health Organ 94: 925-930.

40. World Bank, 2020. Data: Nigeria: GNI Per Captia, Atlas Method. Available at: https://data.worldbank.org/country/nigeria. Accessed October 18, 2020.

41. GBD 2017 DALYs, HALE Collaborators, 2018. Global, regional, and national disability-adjusted life-years (DALYs) for 359 diseases and injuries and healthy life expectancy (HALE) for 195 countries and territories, 1990-2017: a systematic analysis for the Global Burden of Disease Study 2017. Lancet 392: 1859-1922. Erratum in: Lancet 2019;393(10190): e44.

42. Onwujekwe O, Uguru N, Etiaba E, Chikezie I, Uzochukwu B, Adjagba A, 2013. The economic burden of malaria on 
households and the health system in Enugu state southeast Nigeria. PLoS One 8: e78362.

43. Wiseman V, Hawley W, Ter Kuile F, Phillips-Howard P, Vulule J, Nahlen B, Mills A, 2003. The cost-effectiveness of permethrintreated bed nets in an area of intense malaria transmission in western Kenya. Am J Trop Med Hyg 68: 161-167.

44. Alonso S, Chaccouri CC, Elobolobo E, Nacima A, Candrinho B, Saifodine A, Saute F, Robertson M, Zullinger R, 2019. The economic burden of malaria on households and the health system in a high transmission district of Mozambique. Malar $J$ 18: 360.

45. National Population Commission (NPC), ICF International, 2013. Nigeria Demographic and Health Survey. Abuja, Nigeria, and Rockville, MD: NPC and ICF International.

46. National Population Commission (NPC) [Nigeria], ICF International, 2019. Nigeria Demographic and Health Survey 2018. Abuja, Nigeria, and Rockville, MD: NPC and ICF.

47. White M, Conteh L, Cibulskis R, Ghani A, 2011. Costs and costeffectiveness of malaria control interventions-a systematic review. Malar J 10: 337.

48. Murray GPD et al., 2020. Barrier bednets target malaria vectors and expand the range of usable insecticides. Nat Microbiol 5: 40-47.

49. World Health Organization, 2015. Evidence Review Group Meeting Report: Conditions for Use of Long-Lasting Insecticidal Nets
Treated with a Pyrethroid and Piperonyl Butoxide. Geneva, Switzerland: WHO.

50. Protopopoff N, 2017. Evaluation of Novel Long Lasting Insecticidal Net and Indoor Residual Spray Product. Available at: https://clinicaltrials.gov/ct2/show/NCT02288637. Accessed March 30, 2020.

51. Staedke SG et al., 2020. Effect of long-lasting insecticidal nets with and without piperonyl butoxide on malaria indicators in Uganda (LLINEUP): a pragmatic, cluster-randomised trial embedded in a national LLIN distribution campaign. Lancet 395: 1292-1303.

52. Protopopoff N, Rowland M, 2018. Accelerating the evidence for new classes of long-lasting insecticide-treated nets. Lancet 16 : 2415-2416.

53. Briet $O$ et al., 2013. Effects of pyrethroid resistance on the cost effectiveness of a mass distribution of long-lasting insecticidal nets: a modelling study. Malar J 12: 77

54. Killeen G, Okumu F, N'Guessan R, Coosemans M, Adeogun A, Awolola S, Etang J, Dabiré R, Corbel V, 2011. The importance of considering community level effects when selecting insecticidal malaria vector control products. Parasit Vectors 4: 160.

55. Swiss Tropical and Public Health Institute, 2015. OpenMalaria. Basel, Switzerland: Swiss Tropical and Public Health Institute. Available at: https://github.com/SwissTPH/openmalaria/wiki. Accessed December 7, 2020. 